\title{
Impact of transmitted CTL escape mutations on replicative capacity and HIV pathogenesis in early infection
}

\author{
J Prince ${ }^{1 *}$, D Claiborne ${ }^{1}$, D Heckerman², J Carlson² , H Prentice ${ }^{3}$, M Schaefer ${ }^{1}$, L Yue ${ }^{1}$, J Mulenga ${ }^{4}$, J Tang ${ }^{3}$, \\ P Goepfert ${ }^{3}$, P Farmer', R Kaslow ${ }^{3}$, S Allen' ${ }^{1}$, E Hunter ${ }^{1}$
}

From AIDS Vaccine 2012

Boston, MA, USA. 9-12 September 2012

\section{Background}

Multiple HLA class I alleles have been shown to influence both HIV-1 transmission and viral load. In transmission pairs, viral loads of acutely infected partners correlate with viral loads $(\mathrm{VL})$ of their chronically infected donors. This correlation becomes highly significant after adjustment for host factors known to modulate viral load. In addition, we have previously demonstrated that transmission of a virus containing multiple HLA-I associated polymorphisms resulted in a lower set point VL in Zambian linked recipients. These studies imply that transmitted viral characteristics play a role in defining early HIV-1 pathogenesis, and it will be important for vaccine development to understand which viral characteristics are responsible for this.

\section{Methods}

We investigated the role that the in vitro replicative capacity (RC) of the transmitted Gag plays in defining HIV-1 clinical parameters, by cloning gag genes from the founder virus in newly infected partners of 149 epidemiologically linked transmission pairs into the subtype C, R5 tropic MJ4 provirus.

\section{Results}

We observed a statistically significant positive correlation between the RC of Gag-MJ4 chimeras and set point VL in seroconverters $(\mathrm{P}=0.013)$. The $\mathrm{RC}$ of the transmitted $\mathrm{Gag}$ also correlated $(\mathrm{P}=0.025)$ to the viral load in the chronically infected donor, pointing to $\mathrm{RC}$ as the major viral characteristic responsible for the link between donor and linked recipient viral loads. The long term clinical benefit associated with the transmission of attenuated viruses was investigated by performing a Kaplan Meier analysis of time to CD4+ count less than 350. Individuals infected with attenuated gag sequences $(\mathrm{RC}<1)$ were delayed in their progression to $\mathrm{CD} 4+$ counts $<350$ compared to high $(\mathrm{RC}>2)$ replicating viruses $(\mathrm{P}=0.034)$.

\section{Conclusion}

Interestingly, this phenomenon seemed to be independent of viral load perhaps highlighting the role that early viral replication may play in defining HIV-1 pathogenesis.

\section{Author details}

${ }^{1}$ Emory University, Atlanta, GA, USA. ${ }^{2}$ Microsoft Research, Los Angeles, CA USA. ${ }^{3}$ University of Alabama Birmingham, Birmingham, AL, USA. ${ }^{4}$ Zambia Emory HIV Research Project (ZHERP), Lusaka, Zambia.

Published: 13 September 2012

\section{doi:10.1186/1742-4690-9-S2-O57}

Cite this article as: Prince et al:: Impact of transmitted CTL escape mutations on replicative capacity and HIV pathogenesis in early infection. Retrovirology 2012 9(Suppl 2):O57.

${ }^{1}$ Emory University, Atlanta, GA, USA

Full list of author information is available at the end of the article

(c) 2012 Prince et al; licensee BioMed Central Ltd. This is an Open Access article distributed under the terms of the Creative Commons 